\title{
SARS-COV-2 IgG and IgM Antibodies in Cancer Patients
}

Asian Pac J Cancer Prev, 22 (8), 2311-2311

\section{Dear Editor}

We would like to share ideas on "Evaluation of Serologic Changes of IgG and IgM Antibodies Associated with SARS-COV-2 in Cancer Patients: A Cohort Seroprevalence Study (Arab et al., 2021). Arab et al., (2021) concluded that "in the present study asymptomatic cancer patients revealed $17 \%$ seropositivity, approximately equal to the general population of the same age, sex, geographic region, and epidemic status." We agree that there is a risk of disease transmission from cancerous patients with asymptomatic COVID-19. Nevertheless, it should discuss on the efficacy of the diagnostic test for assessment of infection. In the present report, the Pishtaz Teb SARS-CoV-2 ELISA kit is used but there is no data on diagnostic property of the test. There is extremely limited information on the Pishtaz Teb SARS-CoV-2 ELISA kit in international literature. Different types of ELISA tests targeting at different targets can result in different sensitivity and specificity (Vengesai et al., 2021; Huber et al., 2021). False positive is also a possible problem (Vengesai et al., 2021; Huber et al., 2021). For further generalization of the finding for disease control plan in any setting, it is necessary to have a validation on diagnostic property of the diagnostic test.

\section{References}

Arab M, Teymoordash SN, Talayeh M, et al (2021). Evaluation of serologic changes of IgG and IgM antibodies associated with SARS-COV-2 in cancer patients: A Cohort Seroprevalence Study. Asian Pac J Cancer Prev, 22, 1667-70.

Huber T, Steininger P, Irrgang P, et al (2021). Diagnostic performance of four SARS-CoV-2 antibody assays in patients with COVID-19 or with bacterial and non-SARS-CoV-2 viral respiratory infections. Eur J Clin Microbiol Infect Dis, 9, 1-15. doi: 10.1007/s10096-021-04285-4. Online ahead of print.

Vengesai A, Midzi H, Kasambala M, et al (2021). A systematic and meta-analysis review on the diagnostic accuracy of antibodies in the serological diagnosis of COVID-19. Syst Rev, 10, 155.

\section{Rujittika Mungmunpuntipantip ${ }^{1 *}$, Viroj Wiwanitkit ${ }^{2}$}

${ }^{1}$ Private Academic Consultant, Bangkok Thailand. ${ }^{2}$ Honorary Professor, Dr DY Patil University, Pune, India. *For Correspondence: rujittika@gmail.com 